\title{
THE
}

\section{Susceptibility calculations for alternating antiferromagnetic chains}

Jill C. Bonner

University of Rhode Island

H. W. J. Blöte

J. W. Bray

I. S. Jacobs

Follow this and additional works at: https://digitalcommons.uri.edu/phys_facpubs

Terms of Use

All rights reserved under copyright.

\section{Citation/Publisher Attribution}

Bonner, J. C., Blöte, H. W.J., Bray, J. W., \& Jacobs, I. S. (1979). Susceptibility calculations for alternating antiferromagnetic chains. J. Appl. Phys., 50, 1810-1812. doi: 10.1063/1.327177

Available at: http://dx.doi.org/10.1063/1.327177

This Article is brought to you for free and open access by the Physics at DigitalCommons@URI. It has been accepted for inclusion in Physics Faculty Publications by an authorized administrator of DigitalCommons@URI. For more information, please contact digitalcommons-group@uri.edu. 


\title{
A|P| $\left.\right|_{\text {Applied Physics }} ^{\substack{\text { Journal of } \\ \text { Ans }}}$
}

\section{Susceptibility calculations for alternating antiferromagnetic chains}

\author{
J. C. Bonner, H. W. J. Blöte, J. W. Bray, and I. S. Jacobs
}

Citation: Journal of Applied Physics 50, 1810 (1979); doi: 10.1063/1.327177

View online: http://dx.doi.org/10.1063/1.327177

View Table of Contents: http://scitation.aip.org/content/aip/journal/jap/50/B3?ver=pdfcov

Published by the AIP Publishing

\section{Articles you may be interested in}

1D antiferromagnetism in spin-alternating bimetallic chains

J. Appl. Phys. 67, 6001 (1990); 10.1063/1.346043

Improved calculation of nonlinear electric susceptibilities of conjugated hydrocarbon chains

J. Chem. Phys. 68, 3481 (1978); 10.1063/1.436256

Calculation of linear and nonlinear electric susceptibilities of conjugated hydrocarbon chains

J. Chem. Phys. 67, 2935 (1977); 10.1063/1.435258

Analytical Treatment of Excluded Volume. III. Alternative Calculations of the Contacts Density in Chains

J. Chem. Phys. 49, 1599 (1968); 10.1063/1.1670284

Alternating Antiferromagnetic Heisenberg Linear Chains

J. Appl. Phys. 39, 969 (1968); 10.1063/1.1656348

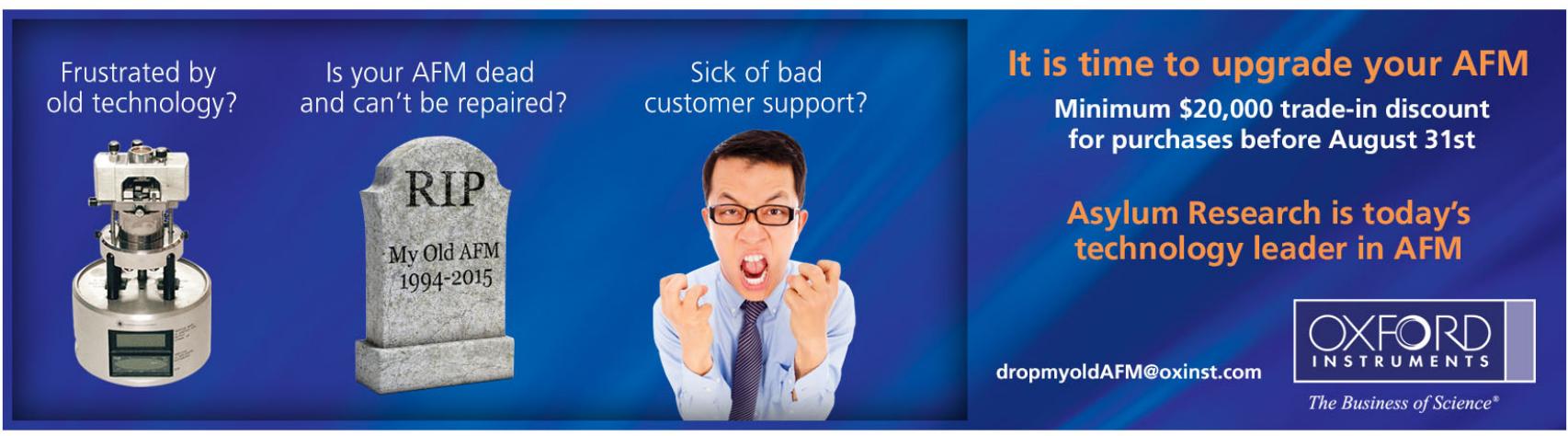




\title{
Susceptibility calculations for alternating antiferromagnetic chains $^{(\mathbf{a})}$
}

\author{
J. C. Bonner ${ }^{(\mathfrak{b})}$ \\ Physics Dept., University of Rhode Island, Kingston, Rhode Island 02881 \\ H. W. J. Blöte(c) \\ Kamerlingh Onnes Laboratorium, Leiden, Netherlands \\ J. W. Bray and I. S. Jacobs \\ General Electric Corp. Res. and Dev., Schenectady, New York 12301
}

\begin{abstract}
Earlier work of Duffy and Barr consisting of exact calculations on alternating antiferromagnetic Heisenberg spin-1/2 chains is extended to longer chains of up to 12 spins, and subsequent extrapolations of thermodynamic properties, particularly the susceptibility, are extended to the weak alternation region close to the uniform limit. This is the region of interest in connection with the recent experimental discovery of spin-Peierls systems. The extrapolated susceptibility curves are compared with corresponding curves calculated from the model of Bulaevskii, which has been used extensively in approximate theoretical treatments of a variety of phenomena. Qualitative agreement is observed in the uniform limit and persists for all degrees of alternation, but quantitative differences of about $10 \%$ are present over the whole range, including the isolated dimer limit. Potential application of the new susceptibility calculations to experiment is discussed.
\end{abstract}

PACS numbers: $75.40 . \mathrm{Fa}, 75.30 . \mathrm{Kz}$

\section{INTRODUCTION}

Quasi-one-dimensional systems continue to attract attention. In addition to the uniform or regular chain, the dimerized (alternating) chain, in which the coupling alternates in magnitude, is important. Specifically the Hamiltonian for an $\mathrm{N}$-spin chain in an applied magnetic field, $H$, is

$$
H=2 J_{1} \sum_{i=1}^{N / 2} \vec{S}_{2 i} \cdot \vec{S}_{2 i+1}+2 J_{2} \sum_{i=1}^{N / 2} \vec{S}_{2 i-1} \cdot \vec{S}_{2 i}-g \mu_{B} H \sum_{i=1}^{N} S_{i}^{z}
$$

where $J_{1}$ and $J_{2}$ are the alternating exchange couplings along the chain $\left(\mathrm{J}_{1}>\mathrm{J}_{2}\right.$, say), assumed to be antiferromagnetic since this is the case of interest. We consider only spin $S=1 / 2$.

In the famous Peierls transition for a half-filled band system, a conducting, regular chain distorts below some transition temperature to an insulating, dimerized chain. Below the transition, some experimental Peierls systems may be described by Hamiltonian (1) to a good approximation if the electrons are well localized. If the electrons retain some mobility, a very closely related Hamiltonian, the dimerized Hubbard model, may be more appropriate. The magnetic analogue of the Peierls transition is tre spin-Peier1s transition [1,2] which occurs only between insulating phases. Here a system of uniform, quasi-one-dimensional $S=1 / 2$, linear Heisenberg antiferromagnet 1c chains in a three-dimensional phonon field distorts to become a system of alternating linear Heisenberg antiferromagnetic chains, well described by Hamiltonian (1), with temperaturedependent alternation [2]. Experimental evidence for many similar phase transitions (first or second order) has stimulated renewed interest in alternating antiferromagnetic chains [3].

The altemating chain is also interesting to physical chemists, particularly those working in the area of spin exciton theory [4]. A review of early approximate theoretical treatments of Hamiltonian (1) is given by Duffy and Barr [5] and others [6]. Duffy and Barr use a numerical approach involving finite chain calculations of up to ten spins. They concentrate primarily on the strongly dimerized regime, where $\mathrm{J}_{1}$ is appreciably greater than $\mathrm{J}_{2}$. It is clear from their work that the best approximate closed form approach is the Hartree-Fock calculation of Bulaevskif [7]. This simple and elegant approximate model has subsequently proved very popular in a variety of applications; to charge-transfer salts $[1,8]$, to spin-dynamics [9], and to the uniform Heisenberg chain [10], where it gives a surprisingly good qualitative account of the thermodynamics [11]. We have extended the work of Duffy and Barr by calculating exactly the properties of alternating chains of up to 12 spins, and extending the extrapolations from the dimer $1 \mathrm{imit}$ all the way to the uniform limit. Hamiltonian (1) may be rewritten as

$H=2 \mathrm{~J}_{1} \sum_{i=1}^{\mathrm{N} / 2}\left\{\overrightarrow{\mathrm{S}}_{2 i} \cdot \overrightarrow{\mathrm{S}}_{2 i+1}+\alpha \mathrm{S}_{2 i-1} \cdot \overrightarrow{\mathrm{S}}_{2 i}\right\}-g \mathrm{H}_{B} \mathrm{H} \sum_{i=1}^{\mathrm{N}} \mathrm{S}_{i}{ }^{z}$

where $\alpha=J_{2} / J_{1}$ is a parameter which conveniently measures the degree of alternation. $\alpha=0$ corresponds to the isolated dimer (spin-pair) system and $\alpha=1$ corresponds to the uniform limit. We have examined the ground state energy per spin, the energy gap between the (singlet) ground state and the band of first excited (triplet) states, and static thermodynamic properties such as the susceptibility and specific heat, all as a function of alternation parameter $\alpha$. Our goal has been to compare the finite chain extrapolations with the Bulaevskii results, which have been employed so extensively, in order to determine their qualitative and quantitative accuracy.

\section{RESULTS}

Details of our results for the ground state energy as a function of alternation, $\varepsilon=E_{0} / J_{1} \mathrm{~N}=$ $\varepsilon(\alpha)$, will be presented elsewhere $[6,12]$. In brief, our extrapolations are in fair agreement with Bulaevskif [7] but in excellent agreement with recent work of Cross and Fisher [13] involving an extension of the Luther-Pesche1 continuum spin-1/2 mode1. [14] to alternating or dimerized systems. A 


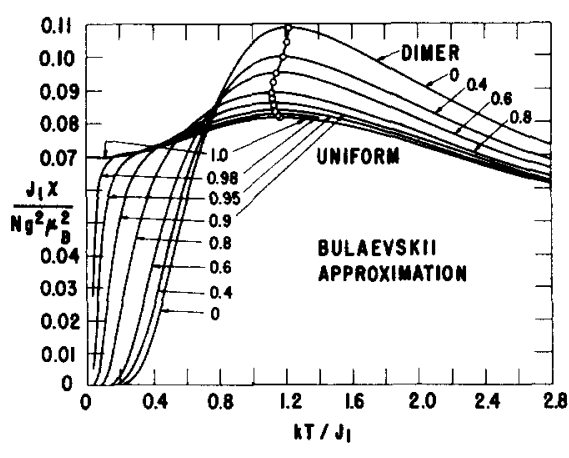

Fig. 1 Antiferromagnetic chain susceptibility vs. Lemperature, for various alternations.

brief account of our results for the energy gap has already been presented [15]. Our extrapolations demonstrate that an excitation energy gap exists for all $\alpha<1$, i.e., for all non-zero degree of alternation. This result is in contrast with exact calculations for the corresponding classica1 $(S=\infty)$ alternating antiferromagnetic Heisenberg chain, which has acoustic mode (i.e., gapless) excitations only, and with a recent Green's function theory [16]. Quantitatively, our results are in fairly good agreement with Bulaevskii [7]. Exact comparison is difficult near the uniform limit because of uncertainty in the numerical extrapolations. This uncertainty is not, however, sufficiently large to cast doubt on our conclusion that an energy gap does indeed exist for $\alpha<1$. The rather close agreement of our gap calculations and those of Bulaevskil is useful in performine the susceptibility extrapolations.

Results for the specific heat are available [6], and may be useful in connection with the analysis of families of fnorganic linear chain systems, some of which appear to have an alternating character. These systems are of interest in coordination chemistry [17]. Magnetic specific heat calculations are not, however, very useful in connection with basically organic Peierls and spin-Peierls type systems because of difficulties in subtracting the very large lattice specific heat of such systems [18].

Hence, for the remainder of this paper, we concentrate on an analysis of the susceptibility per spin as a function of alternation parameter $\alpha$. In Fig. 1 we show zero-field susceptibility curves, calculated exactly on the basis of information in the paper of Bulaevskif [7]. Note that the susceptibility curves vanish exponentially as temperature, $T \rightarrow 0$, for all $\alpha<1$. This is in accordance with the presence of an excitation energy gap discussed above. In the uniform limit, $\alpha=1$, the ground and excited states approach one another as $1 / \mathrm{N}$. The gap therefore vanishes in the thermodynamic limit, and a finite, non-zero susceptibility is obtained in the limit $\mathrm{T} \rightarrow 0$. At finite temperatures, all the susceptibility curves show rounded maxima characteristic of one-dimensional systems. The locus of susceptibility maxima is shown

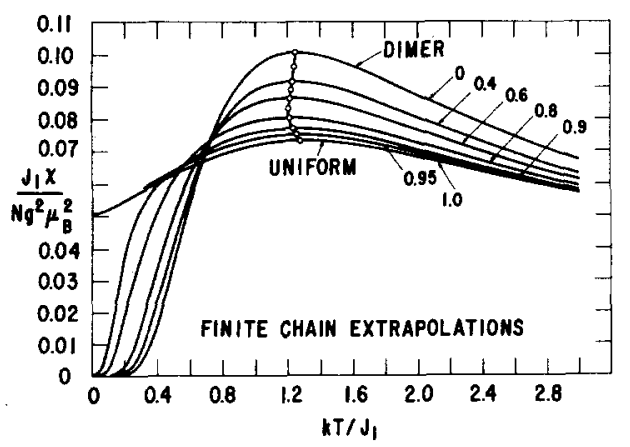

Fig. 2 Antiferromagnetic chain susceptibility vs. temperature, for various alternations.

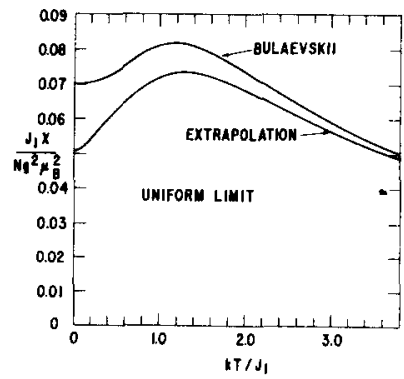

Fig. 3 Comparison of antiferromagnetic chain susceptibility curves in the uniform limit.

as the solid curve through the circles. The variation is rather small (in percentage terms) as $\alpha$ varies from 0 to 1 .

In Fig. 2, we show the corresponding susceptibility curves obtained by extrapolation from finite chain susceptibilities (except for the case $\alpha=0$ which is trivial to calculate exactly: It is the wellknown singlet-triplet spin-1/2 dimer). The alternation $\alpha$ values are the same as for the Bulaevskii calculations, except that $\alpha=0.98$ is omitted because extrapolation becomes very uncertain at very low temperatures for $\alpha$ very close to 1 . Clearly, the qualitative similarities between the Bulaevskii curves and the extrapolations are quite striking. Even the locus of the susceptibility maxima (shown in Fig. 2 as the solid Iine through circles) has the same behavior. This result demonstrates a justification for use of the Bulaevskii approximation in previous treatments of the spinPeierls transition and other phenomena. However, quantitatively, the two sets of curves show a difference which persists for all values of the alternation parameter. For example, the peak values of the Bulaevskil curves are consistently higher than the extrapolations, varying from about $8 \%$ at the dimer limit to about $11 \%$ at the uniform 1 imit. The zeropoint susceptibility for the uniform limit, which is $\left(J x_{0} / \mathrm{Ng}^{2} \mu_{B}\right)=1 /[2(\pi+4)] \simeq 0.0700$ in the Bulaevskii calculation, is about $30 \%$ higher than exact result $\left(\mathrm{J} x_{0} / \mathrm{Ng}^{2} \mu_{B}^{2}\right)=1 / 2 \pi^{2} \simeq 0.050661$ [19]. In the uniform limit, the position of the maximum differs by about $9 \%$ from our numerical estimates. Fig. 3 is a comparison of the Bulaevskii calculation for a uniform chain [10] and extrapolations [20].

That the Bulaevskii curve is significantly quantitatively different from the exact result in the dimer limit may be worthy of further comment. The Bulaevskii approximation fails to preserve the spin rotational symetry of the Heisenberg Hamiltonian. The Bulaevskii chain therefore resembles a linear chain with some transverse anisotropy, i.e., an XYHeisenberg linear chain. The susceptibility in the dimer limit $\left(\mathrm{J}_{2}=0\right)$ is given exactly by the expression

$$
\mathrm{J}_{1} \mathrm{X} / \mathrm{Ng}^{2} \mu_{\mathrm{B}}^{2}=\left(\mathrm{J}_{1} / \mathrm{kT}\right)\left[3+\exp \left(2 \mathrm{~J}_{1} / \mathrm{kT}\right)\right]^{-1} \text {; }
$$

and in the Bulaevskii approximation by

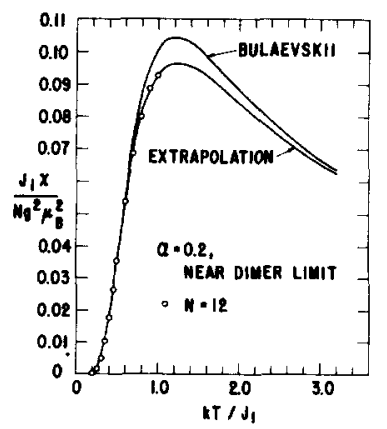

Fig. 4 Comparison of antiferromagnetic chain susceptibility curves near the dimer limit. 


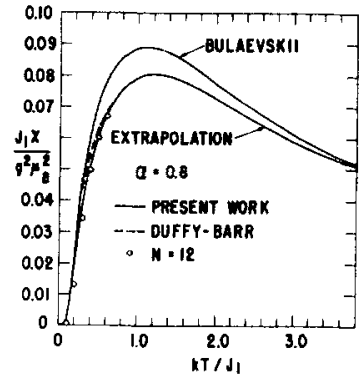

Fig. 5 Comparison of antiferromagnetic chain susceptibility curves for intermediate alternation.

$$
\mathrm{J}_{1} X / \mathrm{Ng}^{2} \mu_{B}^{2}=(1 / 2)\left[1+\left(2 \mathrm{kT} / \mathrm{J}_{1}\right) \cosh ^{2}(\phi / 2 \mathrm{kT})\right]^{-1}
$$

where $\phi=J_{I}[1+\tanh (\phi / 2 \mathrm{kT})]$. Expressions (3) and (4) agree in the low temperature and high temperature limits, but differ in the mid-range.

In Figs. 4 and 5, for $\alpha=0.2$ and $\alpha=0.8$, respectively, we compare the Bulaevskif and extrapolated susceptibilities for intermediate alternation. We should mention that the earlier Duffy-Barr susceptibility extrapolations showed good convergence for $\alpha<0.6$, and our calculations on longer chains do not significantly differ from their results. However, for $\alpha=0.8$ there is some discrepancy. For $\mathrm{kT} / \mathrm{J}$ $\lesssim 0.8$, the Duffy-Barr estimate (which exists only down to $\mathrm{kT} / \mathrm{J}_{1}$ ح0.3) is a few percent higher than our extimate. (We show also the points for finite $\mathrm{N}=12$ in this region, in Fig. 5.)

For a close to the uniform limit, the energy gap is relatively small and this gives rise to a "shouldering" effect. For $\mathrm{kT} / \mathrm{J}_{1}$ large compared with the energy gap $\left(\Delta E / J_{1}\right)$, the susceptibility tends to behave as though it has a finite, non-zero value at $T=0$ (as $1 \mathrm{~s}$ the case for the uniform 1imit). Only when the temperature is comparable to the gap, does the susceptibility "notice" the existence of a gap, and turn downwards rather abruptly, going exponentia11y to zero as $\mathrm{T} \rightarrow 0$. This "shouldering" effect is characteristic (and therefore diagnostic) of weak alternation, and may even be mistaken for a spinPeierls transttion. The effect is rather more apparent for the Bulaevskii curves of Fig. 1 than for the extrapolations of Fig 2, but extrapolation uncertainties make this conclusion rather tentative.

In Fig. 6, the variation with $\alpha$ of the value of the susceptibility at the peak is shown for the two cases of the Bulaevskil approximation, $\mathrm{Fig}, 1$, and the finite chain extrapolations, Fig. 2. Both curves have a similar shape, the Bulaevskii curve lying above the accurate, extrapolated curve by an amount which remains nearly constant as a function of $\alpha$, and therefore the difference changes slightly in percentage terms, from 8 to $11 \%$, as noted earller. In the uniform limit, the peak value of the susceptibility deduced by extrapolation is $\left(\mathrm{Jx} \max _{\max } / \mathrm{Ng}^{2} \mu_{\mathrm{B}}{ }^{2}\right) \simeq 0.07346$ at $\mathrm{kT} \max _{\max } / \mathrm{J}$

$\simeq 1.28$. The Bulaevskii maximum is $\left(\mathrm{J} \chi_{\max } / \mathrm{Ng}^{2} \mu_{B}{ }^{2}\right)$

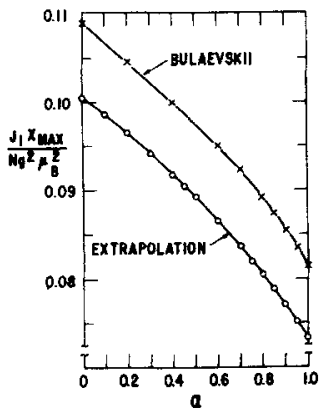

Fig. 6 Variation of peak susceptibility with alternation.
$=0.0816$ at $\mathrm{kT}_{\text {max }} / \mathrm{J}=1.168$. (See Fig. 3.) It may be noted that maxe lower curve in Fig. 6 may be useful for rough experimental analysis. Given a measured susceptibility curve suspected to correspond to an alternating chain, the degree of alternation $(\alpha)$ can be estimated as follows: Determine $J_{I}$ from the temperature corresponding to $x_{\max }$ (since $\mathrm{kT}\left(x_{\max }\right) / 1$

$v_{1.25}$ independent of $\alpha$ ), then convert $x_{\max }$ to reuuced units and read off the value of $\alpha$ using Fig. 6 . This preliminary estimate should be helpful in setting the stage for a more preeise experimentaltheoretical fit.

In summary, we present rather accurate numerical susceptibility calculations for spin-1/2 alternating antiferromagnetic chains in the expectation they will be useful both to experimentalists working in the field of spin-Peierls and generalized Pelerls transitions, and to coordination chemists. More extensive data are available than have been presented in this short report, and are available on request to the authors.

One of us (J.C.B.) would like to thank W. Duffy, Jr., for supplying eigenvalue data at an early stage of the investigations.

\section{REFERENCES}

a) Supported in part (for J.C.B.) by NATO Scientific Research Grant 1338 and NSF grant DMR-77-24136.

b) Fellow of the Institute for Independent Study, Radc11ffe College, Cambridge, MA. 02138.

c) Present address: Univ. of Rhode Island, Kingston.

1. E. Pytte, Phys. Rev. B10, 4037 (1974), and further work cited in ref. 2 .

2. J.W. Bray et a1., Phys. Rev. Lett. 35, 744 (1975); I.S. Jacobs et a1., Phys. Rev. B14, 3036 (1976).

3. E.g., K.A. Penson et al., Phys. Rev. B13, 433 (1976); D.J. Klein et a1., Phys. Rev. B (to appear 1979); J.B. Torrance, Conference on Synthesis and Properties of Low-Dimensional Materials, Ann. NY Acad. Sci., to be published.

4. Z.G. Soos, J. Chem Phys. 46, 4284 (1967).

5. W. Duffy, Jr., and K.P. Barr, Phys. Rev. 165, 647 (1968).

6. J.C. Bonner and H.W.J. BlUte, unpublished work.

7. L.N. Bulaevskit, Sov. Phys.-JETP 17, 684 (1963); Sov. Phys.-Solid State 11, 921 (1 $\overline{1969}$ ).

8. S. Etemad and E. Ehrenfreund, AIP Conf. Proc. 10, 1499 (1973); S.K. Khanna et al., AIP Conf. Proc. 10, 1509 (1973).

9. T. Todani and K. Kawasaki, Progr. Theor. Phys. 50, 1216 (1973).

10. L.N. Bulaevskii, Sov. Phys.-JETP 16, 685 (1963).

11. J.C. Bonner, Thesis, Univ. of London (1968) unpub1ished.

12. J.N. Fields and J.C. Bonner, to be presented at this conference.

13. M.C. Cross and D.S. Fisher, to be published.

14. A. Luther and I. Peschel, Phys. Rev. B12, 3908 (1975).

15. J.C. Bonner, H.W.J. BIBte and J.D. Johnson, J. Phys. (Paris) 39, (Supplement) C6-710 (1978).

16. M. Drawid and J.W. Halley, AIP Conf. Proc. 34, 208 (1976).

17. J.A.C. van Ooijen and J. Reedijk, Inorg. Chim. Acta 25, 131 (1977); V.H. Crawford and W.E. Hatfield, Inorg. Chem. 16, 1336 (1977).

18. J.C. Bonner et al., J. Appl. Phys. 49, 1321 (1978).

19. R.B. Griffiths, Phys. Rev. 133, A768 (1964); C.N. Yang and C.P. Yang, Phys. Rev. 151, 258 (1966).

20. H.W.J.B. has recently performed extrapolations for the susceptibility of the unfform linear Heisenberg antiferromagnet on longer chains, up to $N=13$. However, the new results remain in agreement with earlier work: J.C. Bonner and M.E. Fisher, Phys. Rev. 135, A640 (1964). 